\title{
Le facteur VIII (anti-hémophilique A) recombinant : relation structure/fonction
}

Le facteur VIII, cofacteur glycoprotéique de la coagulation plasmatique, est absent ou non fonctionnel chez les hémophiles de type A. Il est synthétisé sous la forme d'un précurseur de $330 \mathrm{kDa}$ rapidement clivé pour donner une molécule bicaténaire formée d'une chaîne lourde de $220 \mathrm{kDa}$ et d'une chaîne légère de $80 \mathrm{kDa}$ liant le facteur von Willebrand. La partie carboxyterminale de la chaîne lourde (domaine B) peut être variablement clivée sans que l'activité procoagulante soit affectée, ce qui signifie que ce domaine n'est pas indispensable à l'activité du facteur VIII. L'activation biologique est la conséquence de coupures des chaînes lourdes et légères par la thrombine, aboutissant à un trimère actif libéré de son association avec le facteur von Willebrand. Cette forme est sensible à la digestion par la protéine $\mathrm{C}$ activée qui, clivant la partie aminoterminale de la chaîne lourde, inactive le facteur VIII. Des formes complètes ou dépourvues du domaine $B$ du facteur VIII ont été préparées par génie génétique. Ces produits font actuellement l'objet d'essais cliniques ou précliniques.

\section{Nicolas Bihoreau}

\section{ADRESSE ET TIRÉS À PART}

N. Bihoreau : docteur de l'université Paris XI Orsay, responsable du laboratoire de biochimie analytique: département de recherche et développement en biotechnologie. T.M. Innovation, 3, avenue des Tropiques, 91943 Les Ulis, France. 'hémophilie A est la conséquence de l'absence ou d'un dysfonctionnement d'un cofacteur de la cascade de la coagulation: le facteur VIII.

Le facteur VIII dans la cascade de la coagulation

Cette cascade correspond à une suite de réactions enzymatiques, contrôlées par de nombreuses activations et inhibitions (figure 1, p. 1044). Une de ces réactions est la protéolyse du fac- teur $\mathrm{X}$ en facteur $\mathrm{Xa}$, qui fait intervenir un complexe comprenant l'enzyme (le facteur IXa), un cofacteur (le facteur VIII activé), des ions calcium et des phospholipides. Cette activation du facteur $\mathrm{X}$ est accélérée environ 200000 fois par la présence du facteur VIII, préalablement activé par la thrombine, le facteur $\mathrm{Xa}$ ou le facteur IXa. Le facteur $\mathrm{Xa}$ ainsi formé coupe la prothrombine en thrombine en présence du cofacteur $\mathrm{Va}$ (homologue structural du facteur VIII). La thrombine (ou facteur IIa) va transformer à son tour le fibrino- 


\section{RÉFÉRENCES}

1. Toole JJ, Knopf JL, Wozney JM, et al. Molecular cloning of cDNA encoding human antihemophilic factor. Nature 1984 ; $312: 343-7$.

2. Wood WI, Capon DJ, Simonsen CC, et al. Expression of active human factor VIII from recombinant DNA clones. Nature $1984 ; 312: 330-7$.

3. Kaufman RJ, Wasley LC, Dorner AJ. Synthesis, processing and secretion of recombinant human factor VIII expressed in mammalian cells. J Biol Chem 1988; 263 : 6352-62.

4. Dorner AJ, Bole DG, Kaufman RJ. The rclationship of $\mathrm{N}$-linked glycosilation and heavy chain-binding protein association with the sccretion of glycoproteins. J Cell Biol $1987 ; 105: 2665-74$.

5. Vehar GA, Keyt B, Eaton DL, et al. Structure of human factor VIII. Nature $1984 ; 312$ : 337-42.

6. Kane WH, Davie EW. Blood coagulation factor $\mathrm{V}$ and VIII : structural and functional similarities and their relationship to hemorrhagic and thrombotic disorders. Blood $1988 ; 71$ : 539-55.

7. Hornsey VS, Griffin BD, Pepper DS, Miclem LR, Prowse CV. Immunoaffinity purification of factor VIII complex. Thromb Haemost 1987 ; 57 : 102-5.

8. Mcjan O, Fert V, Dclezay M, Dclaagc M, Cheballah R, Bourgois A. Immunopurification of human $\mathrm{FVIII/vWf} \mathrm{complex}$ from plasma. Thromb Haemost 1988; 59: 364-71.

9. Zimmerman TS. Purification of factor VIII by monoclonal antibody affinity chromatography. Semin Hematol 1988 ; 1 : 25-6.

10. Fay PJ, Anderson MT, Chavin SI, Marder VJ. The size of human factor VIII heterodimers and the effect produced by thrombin. Biochim Biophys Acta 1986; 871 : 268-78.

11. Andersson LO, Forsman N, Huang K, et al. Isolation and characterization of human factor VIII : molecular forms in commercial factor VIII concentrate, cryoprecipitate and plasma. Proc Natl Acad Sci USA 1986; $83: 2979-83$.

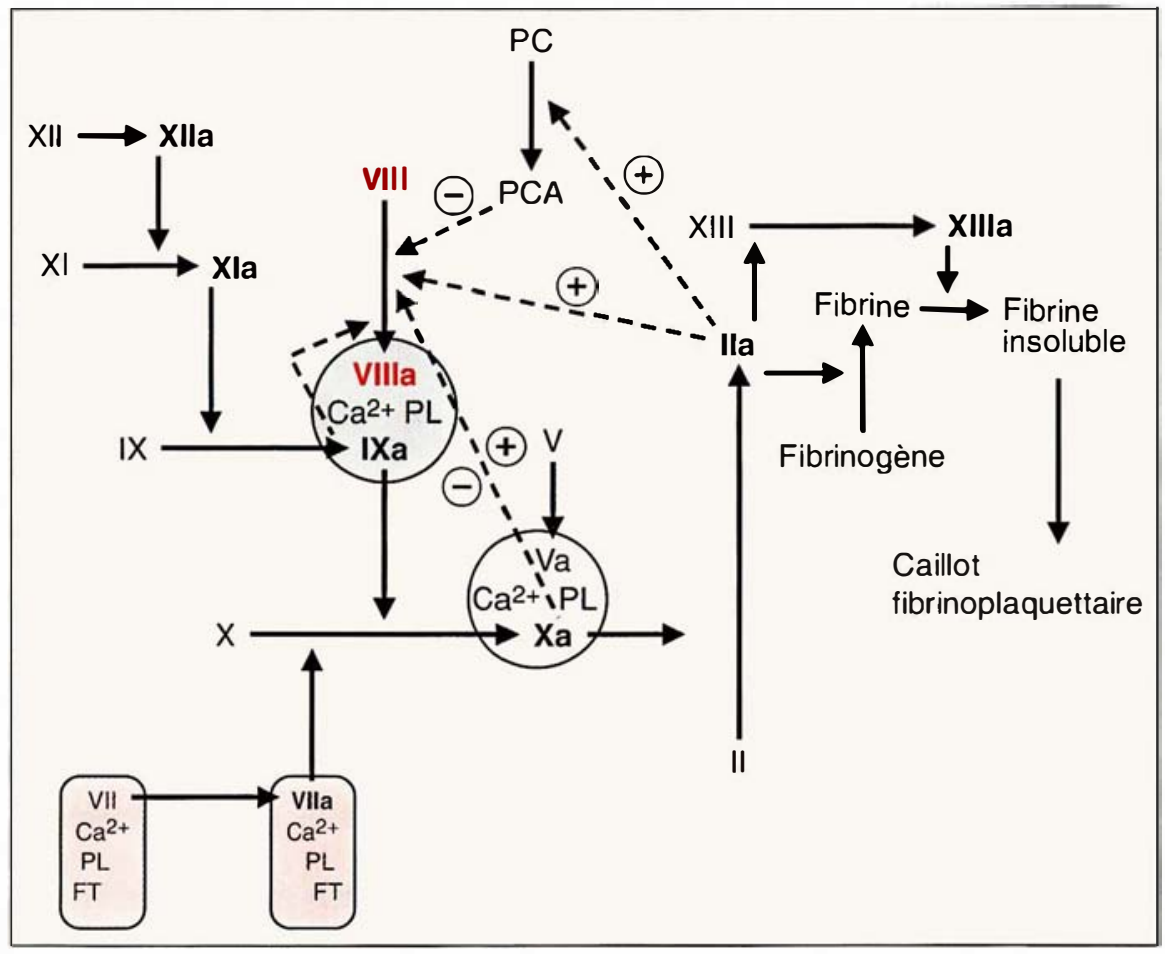

Figure 1. Schéma simplifié de la coagulation. La coagulation comprend plusieurs étapes de conversion d'un zymogène (facteurs XI, XII, VII, etc.) en enzyme active (caractérisée par l'indice " $a »)$. Certaines réactions sont accélérées en présence de cofacteurs (facteurs Va ou VIIla), de phospholipides (PL) et d'ions calcium $\left(\mathrm{Ca}^{2+}\right)$. Les mécanismes d'activation $\oplus$ et d'inactivation $\theta$ sont représentés $(-\rightarrow)$. FT: facteur tissulaire, PCA : protéine $C$ activée.

gène en fibrine et activer le facteur XIII qui lui-même stabilise la fibrine. Les autres substrats de la thrombine sont des inhibiteurs de la coagulation comme la protéine $\mathrm{C}$, qui, sous forme activée, intervient dans l'inactivation du facteur VIII. Enfin, les monomères de fibrine vont former, en liaison avec le facteur XIIIa, un réseau englobant les plaquettes. Ce caillot fibrinoplaquettaire arrête le saignement en bouchant la brèche vasculaire.

\section{Synthèse du facteur VIII}

Le facteur VIII est essentiellement synthétisé dans les cellules hépatiques bien que de l'ARNm de facteur VIII ait été détecté dans d'autres tissus. Le clonage et l'expression de l'ADNc du facteur VIII [1, 2] dans des cellules de mammifère ont permis de proposer un mécanisme de biosynthèse et de maturation de la protéine [3].
L'ADNc code pour un polypeptide de 2351 acides aminés qui perdrait son peptide signal de 19 acides aminés au cours du passage dans le réticulum endoplasmique, où s'effectuerait par ailleurs la glycosylation. Une fraction du facteur VIII fixé à des protéines du réticulum endoplasmique, les BiP (binding protein, [4]), serait dégradée. L'autre partie transiterait vers le Golgi où s'effectueraient, d'une part, les modifications post-traductionnelles (addition des ions sulfates aux résidus tyrosines et des carbohydrates aux acides aminés sérine et thréonine) et, d'autre part, la coupure de la protéine de $330 \mathrm{kDa}$ en une chaîne légère de $80 \mathrm{kDa}$ et une chaîne lourde de 210 kDa. La formation de complexes actifs dépendrait de la présence du facteur von Willebrand qui associe les deux châ̂nes libres. En son absence, les châ̂nes lourde et légère du facteur VIII non associées seraient sécrétées et dégradées [3]. 


\section{Structure du facteur VIII}

L'analyse de la séquence en acides aminés, déduite de l'ADNc humain, a montré que le facteur VIII est constitué de trois domaines structuraux différents: $A, B$ et $C$. Le domaine $\mathrm{A}$, de 330 acides aminés, est présent en trois exemplaires; la région $\mathrm{B}$, unique, possède 983 acides aminés ; et les deux domaines $\mathrm{C}$ contenant 150 acides aminés sont situés dans la partie carboxyterminale du facteur VIII. Ces différents domaines sont arrangés suivant l'ordre A1-A2-B-A3-C1-C2 (figure 2) [5]. Les domaines $\mathrm{A}$ ont entre eux $30 \%$ d'homologie alors que les domaines $\mathrm{C}$ ont entre eux $40 \%$ d'homologie. Le facteur VIII possède 23 résidus cystéines dont 19 sont répartis dans les domaines $\mathrm{A}$ et $\mathrm{C}$ à des positions identiques d'un domaine homologue à l'autre, ce qui suggère la formation de ponts disulfures équivalents dans chacun de ces domaines.

Il existe également $30 \%$ d'homologie entre les domaines $\mathrm{A}$ du facteur $\mathrm{V}$ [6] et du facteur VIII. Le domaine $B$ n'a pas d'homologie connue avec d'autres protéines. Il possède 19 sites potentiels de glycosylation sur les 25 identifiés pour le facteur VIII. Le domaine $\mathrm{C}$ présente une homologie avec des lectines capables de se lier aux phospholipides chargés négativement.

Le facteur VIII est présent dans le plasma en faible concentration $(0,1 \mu \mathrm{g} g / \mathrm{ml})$ et est coupé par de nombreuses sérine protéases. Ces caractéristiques ont rendu difficile la purification de cette protéine. Le facteur VIII a été purifié par de nombreuses techniques et récemment grâce à des méthodes d'immunopurification [7-9]. Celles-ci permettent, en une seule étape, de piéger les différentes formes de facteur VIII actif pour obtenir, avec un rendement élevé, un produit de haute activité spécifique et forte concentration. A partir du produit immunopurifié, différents complexes actifs de facteur VIII ont été séparés et caractérisés $[10,11]$. Ce sont des formes bicaténaires ayant en commun une chaîne de 80 kDa (chaîne légère) et dont l'autre chaîne (chaîne lourde) peut avoir un poids moléculaire compris entre 210 et $90 \mathrm{kDa}$ (figure 2). Les $\mathrm{m} / \mathrm{s} n^{\circ} 10 \mathrm{vol} .8$, décembre 92

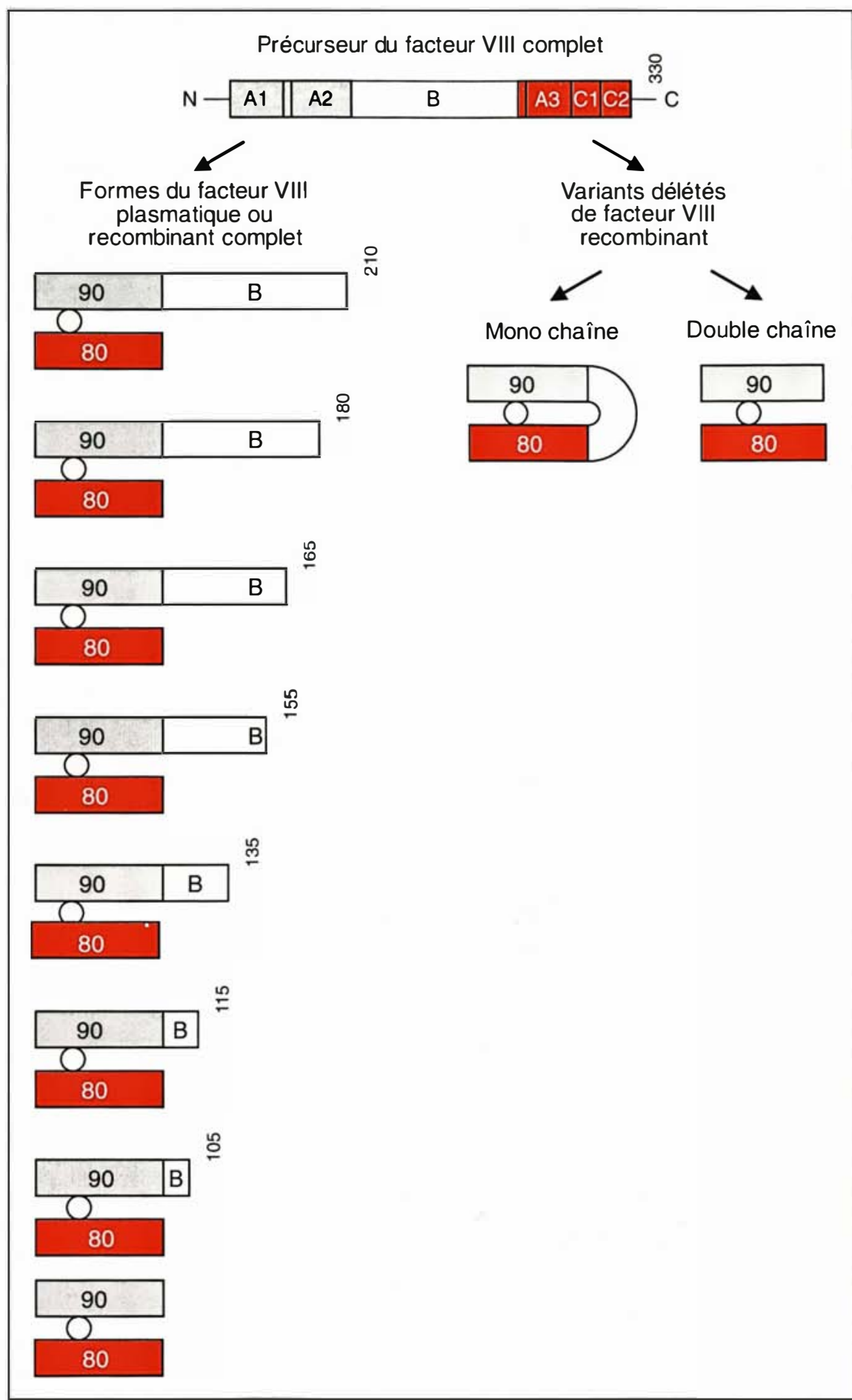

Figure 2. Les différentes formes du facteur VIII. Le facteur VIII complet de $330 \mathrm{kDa}$, comprend trois domaines $A, B$ et $C$. Les différents dimères de facteur VIII, dont la région $B$ est plus ou moins protéolysée, correspondent à l'association d'une chaîne lourde (de 210 à $90 \mathrm{kDa}$ ) avec une chaîne légère (de $80 \mathrm{kDa}$ ), par un cation divalent (O). 


\section{RÉFÉRENCES}

12. Nordfang O, Ezban M, Hansen JJ FVIII subunits : purification and antigenic properties. Thromb Haemost $1987 ; 58$ : 1043-8.

13. Nordfang O, Ezban M. Generation of active coagulation factor VIII from isolated subunits. J Biol Chem 1988; 263 : 1115-8.

14. Fay PJ. Thrombin-activated factor VIIIa is composed of a non covalent $73 / 51$ Kd dimer. Thromb Haemost 1987 ; 58 : 343 (abstr 1241)

15. Lollar P, Parker CG. Subunits structure of thrombine-activated porcine factor VIII. Biochemistry 1989 ; 28 : 666-74.

16. Eaton DL, Wood WI, Eaton D, et al. Construction and characterization of an active factor VIII lacking the central one third of the molecule. Biochemistry $1986 ; 25$ : 8343-7.

17. Toole JJ, Pittman DD, Orr EC, Murtha $P$, Wasley LC, Kaufman RJ. A large region ( $=95 \mathrm{kDa}$ ) of human factor VIII is dispensable for in vitro procoagulant activity. Proc Natl Acad Sci USA 1986; 83 : 5939-42.

18. Brinkhous $\mathrm{KM}$, Sandberg $\mathrm{H}$, Garris $\mathrm{JB}$, et al. Purified human factor VIII procoagulant protein : comparative hemostatic response after infusions into hemophilic and von Willebrand disease dogs. Proc Natl Acad Sci USA 1985 ; 82 : 8752-6.

19. Pittman DD, Kaufman RJ. Proteolytic requirements for thrombin activation of antihemophilic factor (factor VIII). Proc Natl Acad Sci USA 1988 ; 85 : 2429-33.

20. Gitshier J, Kogan S, Levinson B. Tuddenham, EGD. Mutations of factor VIII clcavage sites in hemophilia A. Blood 1988 ; 72 : 1022-8.

21. Fay PJ, Haidaris PJ, Smudzin TM. Reconstitution of factor VIIIa from the isolated A1/A3-C1-C2 dimer and the A2 sub unit. J Biol Chem 1991; 266 : 8957-62.

22. Foster PA, Fulcher CA, Houghten RA Mahoney SG, Zimmerman TS. Localization of the binding regions of a murine monoclonal anti-factor VIII antibody and a human anti-factor VIII alloantibody, both of which inhibit factor VIII procoagulant activity, to aminoacid residues Thr ${ }^{351}$-Ser 365 of the factor VIII heavy protéases responsables du clivage du facteur VIII en deux chaînes lourde et légère et du clivage progressif de la région $\mathrm{B}$ n'ont pas été identifiées à ce jour. Les séquences $\mathrm{N}$-terminales des différentes chaînes lourdes étant identiques [11], celles-ci sont issues de la dégradation progressive de l'extrémité C-terminale de la chaîne lourde de $210 \mathrm{kDa}$ (figure 2).

L'association entre les chaînes lourde et légère du facteur VIII, qui est indispensable à l'activité coagulante [12], se fait par l'intermédiaire d'un ion divalent. La nature de cet ion est inconnue à ce jour. L'activité coagulante est restaurée si les deux chaînes du facteur VIII sont mises en présence d'ions $\mathrm{Mn}^{2+}$ ou $\mathrm{Ca}^{2+}$ [13]. Ces mêmes ions sont indispensables à la réassociation des fragments isolés du facteur $\mathrm{V}$, homologue structural du facteur VIII. Les sites exacts d'interaction du facteur VIII avec les ions divalents ne sont pas connus précisément. Cependant, l'identification de complexes de faible poids moléculaire, de 70-50 kDa, suppose que l'ion divalent associe les sousdomaines homologues A1 et A3 [14].

\section{Rôle de la région $B$}

De multiples arguments prouvent que la région $\mathrm{B}$ n'est pas nécessaire à l'activité procoagulante du facteur VIII. La comparaison des séquences en acides aminés entre les facteurs VIII humain et porcin fait apparaître des différences majeures au niveau de cette région $B$ [15]. Le facteur VIII porcin est néanmoins utilisé efficacement pour le traitement de l'hémophilie A, en substitution du facteur VIII humain, chez les hémophiles ayant des inhibiteurs.

Des protéines recombinantes, obtenues à partir de l'ADNc de facteur VIII délété de la partie codante pour la région $\mathrm{B}$, se sont révélées aussi actives que la molécule de facteur VIII complète $[16,17]$. De plus, toutes les formes bicaténaires naturelles (figure 2) contenant tout ou partie de cette région sont également actives. Brinkous et al. [18] ont montré, chez le chien hémophile, que les complexes actifs de $90-80 \mathrm{kDa}$, sans région $\mathrm{B}$, avaient un temps de demi-vie plus long que le facteur VIII plasmatique avec région $B$. Cette différence de stabilité serait due à une fixation préférentielle du facteur VIII sans région $\mathrm{B}$ à sa protéine porteuse : le facteur von Willebrand. Par ailleurs, la thrombine active in vitro plus rapidement les variants délétés de la région $B$ que le facteur VIII recombinant complet [16]. Ces observations confirment l'influence de la région $B$ dans le processus d'activation du facteur VIII par la thrombine. $\mathrm{Ce}$ domaine B n'est donc pas indispensable à l'activité procoagulante mais contrôlerait l'action d'effecteurs de la cascade de la coagulation.

\section{Activation et inactivation}

Plusieurs protéines interviennent dans la régulation de l'action du facteur VIII. Celui-ci est, d'une part, activé par la thrombine, le facteur Xa et le facteur IXa, et, d'autre part, inactivé par la protéine $\mathrm{C}$ activée. Eaton et al. [16] ont démontré que l'augmentation brutale de l'activité coagulante observée après action de la thrombine était liée à la protéolyse de la chaîne légère et de la chaîne lourde du facteur VIII pour former le complexe activé de 70-50-45 kDa (figure 3).

Pittman et al. [19] ont déterminé, par mutagenèse dirigée, les sites de clivage du facteur VIII par la thrombine. La mutation des arginines en position 740 et 1648 (figure 3) par des isoleucines empêche la protéolyse, qui engendre les polypeptides de 90 et 80 $\mathrm{kDa}$, sans altérer l'activation in vitro. Cela démontre que la coupure de la région $B$ n'est pas une étape préalable à l'activation du facteur VIII. En revanche, lorsque les sites 372 et 1689 du facteur VIII sont mutés [18], le variant n'est plus protéolysé par la thrombine au niveau des sites modifiés et il n'est pas activable, in vitro, par cet effecteur. Ces dernières coupures sont donc indispensables pour l'activation du facteur VIII. De plus, si un seul de ces deux sites est modifié, l'activation n'a toujours pas lieu, ce qui suggère un processus de clivage non séquentiel lors de l'activation pour former le complexe de 70-50-45 kDa.

Enfin, l'hémophilie sévère observée chez certains patients dont la structure du facteur VIII diffère par une substitution de l'arginine 1689 en cystéine [20] ou de l'arginine 372 en 
histidine, confirme l'importance de ces sites de clivage pour l'activité du facteur antihémophilique $\mathrm{A}$ in vivo. La figure 3 montre que la thrombine et le facteur Xa coupent les chaînes lourdes du facteur VIII après l'arginine 740 pour engendrer un polypep- tide de 90 kDa lui-même protéolysé, après le résidu 372, en deux fragments de 50 et $45 \mathrm{kDa}$. Le polypeptide de $50 \mathrm{kDa}$ correspond à l'extrêmité $\mathrm{N}$-terminale de la protéine. La chaîne légère du facteur VIII de $80 \mathrm{kDa}$ est clivée en position 1689

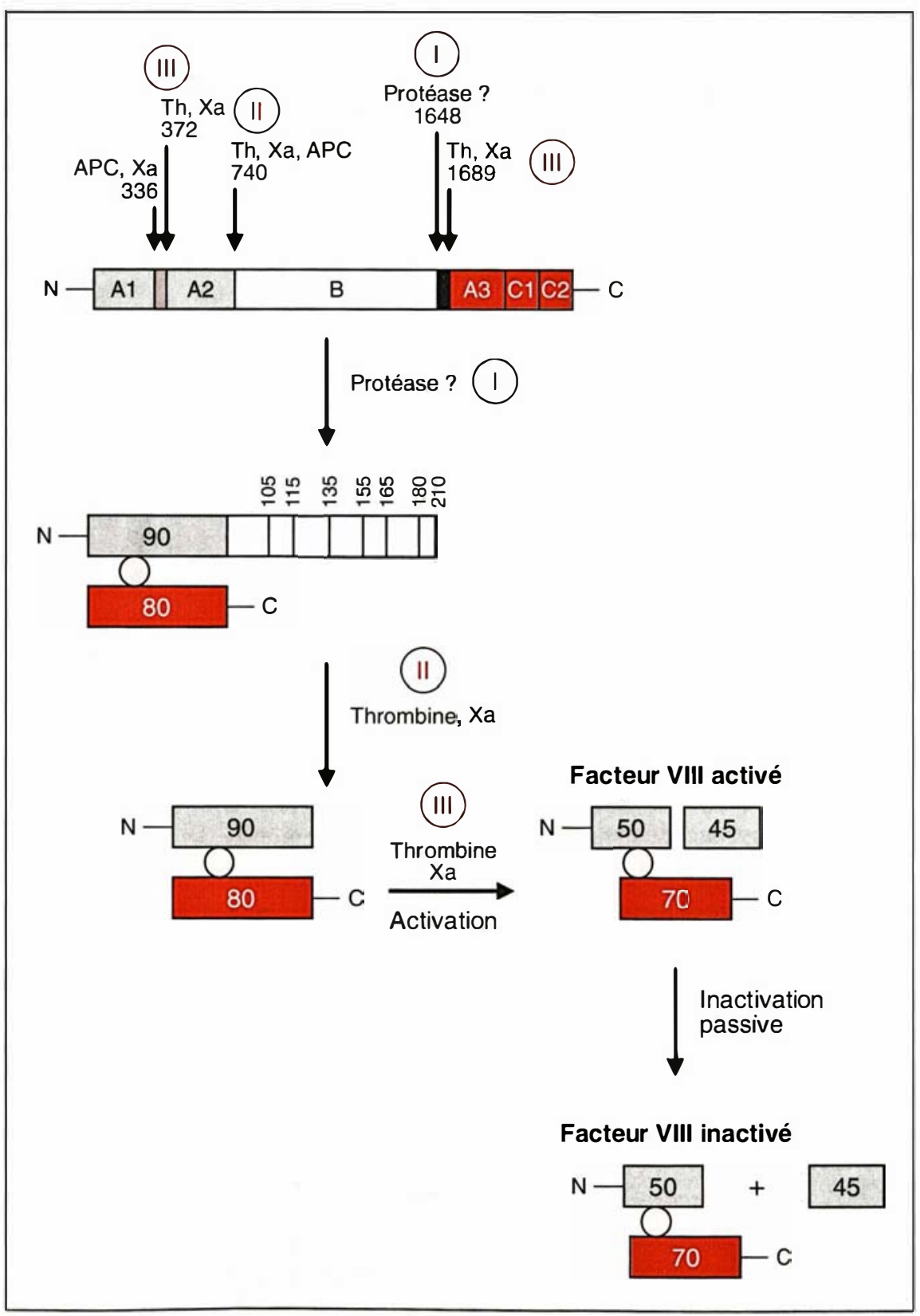

Figure 3. Activation par la thrombine ou le facteur $\mathrm{Xa}$. Les sites de clivage de la thrombine (Th) et du facteur Xa (Xa) sont indiqués $(\rightarrow)$. (I) Protéolyse en position 1648 par une protéase inconnue (?). (II) Protéolyse de la région $B$ après le résidu Arg740 pour former le dimère de 90-80 kDa de chaînes lourde et légère associées par le cation divalent (O). (III) Activation par la thrombine ou le facteur Xa par protéolyse du facteur VIII après l'Arg 372. Les masses moléculaires des fragments qui constituent le facteur VIII activé sont représentés en $k D a$. L'inactivation passive correspond à la dissociation du fragment $C$ terminal de $45 \mathrm{kDa}$ du complexe activé.

$\mathrm{m} / \mathrm{s} n^{\circ} 10 \mathrm{vol}$. 8, décembre 92 par la thrombine et le facteur $\mathrm{Xa}$ pour engendrer une protéine de 70 $\mathrm{kDa}$.

Après formation du complexc activé de 70-50-45 kDa, la phase d'inactivation observée à la suite du contact prolongé avec la thrombine est indépendante de cette protéase et correspondrait plutôt à une dissociation du complexe en un dimère de $70-50 \mathrm{kDa}$ et un fragment de $45 \mathrm{kDa}$ (figure 3). Le facteur Xa modifie aussi les formes activées en protéolysant les polypeptides de 70 et $50 \mathrm{kDa}$ en deux fragments de, respectivement, 67 et $45 \mathrm{kDa}$. Ces clivages entraînent une diminution brutale de l'activité procoagulante. La protéine $\mathrm{C}$ activée se lie à la chaîne légère du factcur VIII pour couper uniquement la chaîne lourde, après les résidus 740 et 336 . La mutation ponctuelle de l'arginine 336 en lysine ou isoleucine provoque une inhibition de l'inactivation par la protéine $\mathrm{C}$ activée [18]. Ce clivage, qui aboutit après dissociation du fragment $\mathrm{A} 2$ à la formation du dimère A1/A3-C1-C2, correspond à l'inactivation du facteur VIII (figure 4, p. 1048). Un trimère actif pcut être reconstitué à partir des deux formes inactives A1/A3-C1-C2 et A2 [21].

La figure 4 montre que les résidus Arg 336 et 372 sont impliqués dans l'activation et l'inactivation du facteur VIII. La région comprise entre ces deux résidus, qui contient 15 résidus acides aspartique et glutamique pour seulement quatre résidus lysine et arginine, doit donc jouer un rôle fonctionnel important [22]. De même, la partie $\mathrm{N}$-terminale de la chaîne légère du facteur VIII correspond à une région très acide (avec également 15 résidus acides aspartique et glutamique pour quatre résidus lysine et arginine). La protéolyse du polypcptide de $80 \mathrm{kDa}$ par la thrombine et le facteur Xa après la sérine 1689 permet de libérer ce fragment acide. Cette deuxième région acide a égalcment un rôle fonctionnel important puisqu'elle intervient dans la liaison du facteur VIII au facteur von Willebrand (figure 4).

\section{Le facteur von Willebrand}

Le facteur VIII est stabilisé par l'association avec une autre glycoprotéine plasmatique, le facteur von Wil- 
lebrand. L'expression de facteur VIII recombinant dans des cellules de mammifères a montré que la protéine synthétisée en absence de facteur von Willebrand est sécrétée sous forme de deux chaînes séparées, très exposées aux attaques enzymatiques. Son rôle dans l'association des chaînes légère et lourde du facteur VIII pour former un complexe actif a été confïmé par des expériences de réassociation in vitro des différentes chaînes libres [23].

Une étude réalisée in vivo [24] sur des chiens hémophiles après injection de facteur VIII recombinant complet ou

\section{RÉFÉRENCES}

23. Fay PJ. Reconstitution of human factor VIII from isolated subunits. Arch Biochem Biophys 1988 ; 262 : 525-31.

24. Giles AR, Tinlin $\mathrm{S}$, Hoogendoorn $\mathrm{H}$ Fourncl MA, Ng P, Pancham N. In vivo characterization of recombinant factor VIII in a canine model hemophilia A (factor VIII deficiency). Blood 1988 ; 72 : 335-9.

25. Meulien P, Faure T, Mischler F, et al. A new recombinant procoagulant protein derived from the cDNA encoding human factor VIII. Prot Eng 1988; 2 : 301-6.

26. Foster PA, Fulcher CA, Houghten RA Zimmerman TS. An immunogenic region within residucs $\mathrm{Val}^{167(0)}-\mathrm{Glu}^{1684}$ of the fac tor VIII light chain induces antibodies which inhibit binding of factor VIII to von Willebrand factor. J Biol Chem 1988; 263 5230-4.

27. Hamer RJ, Kocdam JA, Becser-Visser NH, Sixma JJ. The effect of thrombin on the complex between factor VIII and von Willebrand factor. Eur $J$ Biochem 1987 ; 167 : 253-9.

28. Leyte A, van Schijndel HB, Nichrs C Sulf ation of Tyr ${ }^{1680}$ of human blood coagulation factor VIII is essential for the interaction of factor VIII whith von Willebrand factor. J Biol Chem 1991; 266 : 740-6.

29. Lollar P, Hill-Eubanks DC, Parker CG Association of the factor VIII light chain with von Willebrand factor. $J$ Biol Chem $1988 ; 263: 10451-5$

30. Hill-Eubanks DC, Parker CG, I ollar P Differential protcolytic activation of factor VIII-von Willebrand factor complex by thrombin. Proc Nall Acad Sci USA $1989 ; 86$ : 6508-12.

31. Bihoreau N, Paolantonacci P, Bardelle $C$, et al. Structural and functional characterization of factor VIII- $\triangle I I$, a new recombinant factor VIII lacking most of the B domain. Biochem J 1991;277: 23-31

32. Krishnan S, Kolbc HVJ, Lepage P, et al. Thrombin cleavage analysis of a novel antihacmophilic factor variant, factor

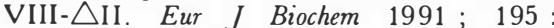

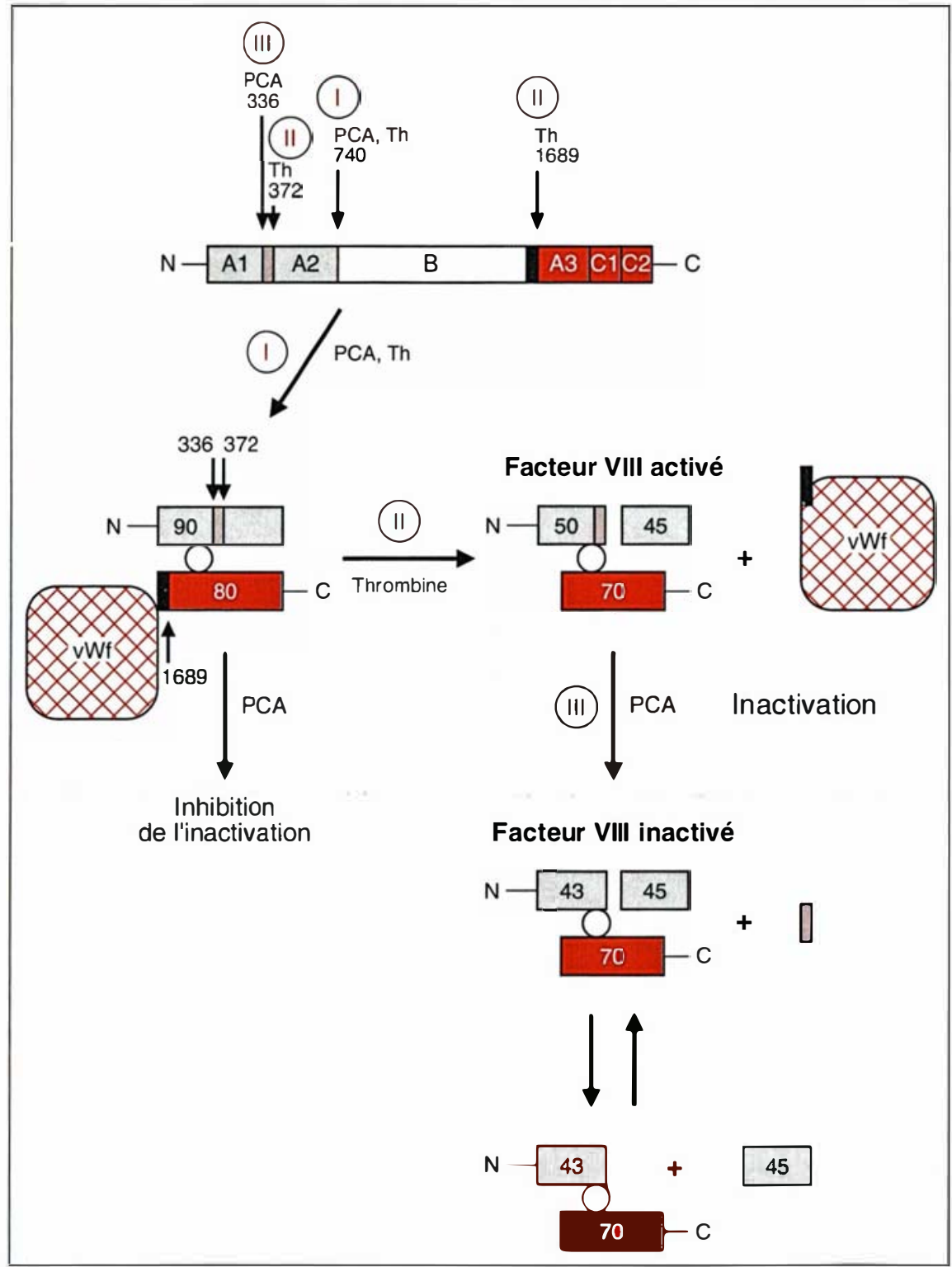

Figure 4. Inactivation par la protéine $\boldsymbol{C}$ activée. Les différents sites de clivage sont représentés $(\rightarrow)$. (I) Clivages en position 740 et 1648 et formation du facteur VIII de 90-80 kDa. Le facteur von Willebrand (vWf) est associé au facteur VIII par la région acide (口) N-terminale de la chaîne légère. (II) Le vWf est séparé du facteur VIII par protéolyse de la région acide (口). (III) Grâce à la perte du vWf, l'inactivation par la protéine $C$ activée (PCA) s'effectue par coupure après le résidu Arg 336. Les poids moléculaires des fragments qui constituent le facteur VIII inactivé sont représentés en $k D a$. Cette inactivation correspond aussi à la perte de la région acide de la chaîne lourde et à la dissociation du fragment C-terminal de $45 \mathrm{kDa}$. 
de facteur VIII plasmatique a prouvé que la formation d'un complexe entre le factcur VIII et le facteur von Willebrand était indispensable au maintien d'unc demi-vie normale.

Sachant que le facteur $\mathrm{V}$ ne se lie pas au facteur von Willebrand, alors qu'il présente une forte homologie avec le facteur VIII, il a été possible d'identifier sur ce dernier plusieurs sites potentiels de fixation au facteur von Willebrand.

Le factcur VIII recombinant obtenu après délétion de la partie codante pour la région $\mathrm{B}$ se lie au facteur von Willebrand de la même manière que la protéine complète [25]. Ces observations ont permis d'éliminer une première hypothèse de liaison sur ce domaine B. De même, la délétion de la région acide de la chaîne lourde provoque la perte de l'activité du mutant alors que celui-ci garde sa lebrand. En revanche, la délétion de la région située entre les acides aminés 1648 et 1689 empêche la fixation du facteur von Willebrand.

Des anticorps dirigés contre un épitope situé entre les acides aminés Val-1670 et Glu-1684 inhibent la fixation du facteur von Willebrand [26]. Enfin, la protéolyse par la thrombine de la chaîne légère $d u$ facteur VIII libère le fragment $\mathrm{N}$ terminal de $7 \mathrm{kDa}$ associé au facteur von Willebrand [27] (figure 4). Ces résultats prouvent que le site de fixation du facteur von Willebrand est situé à l'extrémité $\mathrm{N}$-terminale de la chaîne légère du facteur VIII. capacité de fixer le facteur von Wil-

Celle-ci possède des tyrosines sulfatées en position 1664 et 1680 [28]. L'implication de ces résidus dans la fixation au facteur von Willebrand a été étudiée par mutagenèse dirigée. Une substitution ponctuelle de ces deux résidus engendre une molécule très instable du fait de sa mauvaisc fixation au facteur von Willebrand. Ces résultats suggèrent que les deux tyrosines modifiées sont d'une grande importance pour l'association des deux glycoprotéines. En revanche, la bonne capacité à fixer le facteur von Willebrand de formes délétées du facteur VIII ne possédant pas la tyrosine 1664 [25], indique que la tyrosine 1680 est la plus impliquée dans l'association du factcur VIII au facteur von Willebrand.

Le rôle d'intermédiaire du facteur von Willebrand dans l'association des chaînes légère et lourde du facteur VIII suppose un site de fixation sur chacune de ces chaînes. Bien qu'aucun site n'ait clairement été identifié sur la chaîne lourde du facteur VIII, il semble que le facteur von Willebrand se fixe tout d'abord à la chaîne légère [29] et s'associe ensuite, après action de la thrombine, au fragment C-terminal de $45 \mathrm{kDa}$ de la chaîne lourde du facteur VIII activé [27]. Cela indique la présence d'un site secondairc de fixation au facteur von Willebrand qui serait plus accessible après l'action de la thrombine.

Hill-Eubanks et al. [30] ont étudié l'effet d'une protéase de venin de serpent similaire à la thrombine mais

Tableau I

PURETÉ DES DIFFÉRENTS FACTEURS VIII PLASMATIQUES

\begin{tabular}{|c|c|}
\hline $\begin{array}{c}\text { Les différents facteurs VIII } \\
\text { plasmatiques }\end{array}$ & Actvité spécifique ${ }^{*}(1)$ \\
\hline Plasma frais & 0,016 \\
\hline Cryoprécipité & 0,1 \\
\hline Concentré standard & 1 \\
\hline Produit de pureté intermédiaire & 100 à 200 \\
\hline Produit immunopurifié & 2000 à 3000 \\
\hline Facteur VIII pur & 10000 \\
\hline
\end{tabular}

* L'activité spécifique est mesurée en unités de facteur VIII par milligramme de protéines. $\mathrm{m} / \mathrm{s} n^{\circ} 10$ vol. 8 , décembre 92 qui n'hydrolyse que la chaîne lourde du factcur VIII. La présence de facteur von Willebrand sur la chaîne légère du factcur VIII inhibe l'action de cette protéasc alors que la thrombine active normalement le facteur VIII. Par ailleurs, l'action protéasique de la protéine $\mathrm{C}$ activéc sur la chaîne lourde du factcur VIII est inhibée par la présence de facteur von Willebrand. Ces résultats confirment qu'une première coupure de la chaîne légère par la thrombine permettant de dissocier le facteur VIII du facteur von Willebrand cst indispensable à la protćolyse ultérieure de la chaîne lourde par la thrombine ou par la protéine $\mathrm{C}$ activćc pour l'activation ou l'inactivation du factcur VIII (figure 4).

\section{Quels facteurs antihémo-} philiques $A$ pour demain?

L'histoire du traitement de l'hémophilic A est jalonnéc par l'utilisation de produits d'origine plasmatique contenant du facteur VIII à des taux de plus en plus ćlevés (Tableau I). Cette évolution vers des produits de plus haute purcté s'est enrichie aussi de l'introduction de traitements inactivant les virus qui garantissent l'innocuité des protéines naturelles. En effet, le don de sang, rémunéré ou non, apporte une part de risques qui ne peuvent être maîtrisés qu'après avoir été subis. Ainsi la contamination d'hémophiles par des produits plasmatiques contenant du VIH n'a pu être stoppée qu'après identification de ce virus en 1983 comme l'agent responsable du SIDA.

De même, le virus de l'hćpatite B, identifié en 1968, contaminait fréquemment ces préparations jusqu'à cc que des méthodes fiables de dépistage, mises en place dans les années 1970 , permettent de rejeter les dons contaminćs.

L'avènement proche des premiers facteur VIII recombinants (Kogenate $\mathrm{ct}$ Recombinate, Tableau II, p. 1050) garantit une innocuitć virale qui ne peut défaillir. D'une part, chaque ccllule productrice provient d'une ligncee de cellules conservées dans l'azote liquide en banques, dont le statut viral est figé et contrôlé. D'autre part, toutes les ćtapes d'élaboration, de la culture cellulaire jusqu'au pro- 


\begin{tabular}{|c|c|c|c|c|}
\hline \multicolumn{5}{|c|}{ Tableau II } \\
\hline LES DIFFÉRENTS FACTEURS VIII RECOMBINANTS & $\begin{array}{c}\text { Groupe } \\
\text { industriel }\end{array}$ & Forme & $\begin{array}{c}\text { Mode de } \\
\text { purification }\end{array}$ & Statut \\
\hline Recombinate & Baxter & Complète & Immunopurif & Prélancement \\
\hline Kogenate & $\begin{array}{c}\text { Cutter Miles } \\
\text { Bayer }\end{array}$ & Complète & Immunopurif & Prélancement \\
\hline FVIII- $\triangle I I$ & T M I & $\begin{array}{c}\text { Délétée } \\
\text { monochaîne } \\
(771-1666)\end{array}$ & $\begin{array}{c}\text { Non } \\
\text { immunopurif }\end{array}$ & Préclinique \\
\hline FVIII : SO & Kabi & $\begin{array}{c}\text { Délétée } \\
\text { bicaténaire } \\
(743-1638)\end{array}$ & Immunopurif & Préclinique \\
\hline
\end{tabular}

duit final, suivent un procédé de fabrication réglementé intégrant un traitement inactivant pour aboutir à un produit pharmaceutique standardisé.

Les normes réglementaires qui fixent à des valeurs seuils très faibles les taux de génome et de protéines cellulaires dans le produit final impliquent en fait que la pureté soit proche de $100 \%$ avant addition d'agents stabilisants.

Les coûts de ces produits recombinants doivent prendre en compte l'amortissement de frais de recherche et de développement longs et coûteux, d'études cliniques et de mise en place de l'outil de production. Le prix de revient industriel dépendra de la productivité cellulaire (exprimée en unités de facteur VIII par million de cellules et par 24 heures) mais aussi du coût du milieu de culture cellulaire. En effet les volumes de milieu utilisés sont considérables et leur coût direct très lié à leur charge en protéines. Un milieu de production chimiquement défini, sans sérum, donc peu chargé en protéines, ne néccssite pas de techniques lourdes et coûteuses de purification (immunopurification, par exemple) par rapport aux méthodes de chromatographie classiques (Tableau II).

Près de dix ans seront écoulés entre le clonage en 1984 du gène du factcur VIII humain et le lancement des facteurs VIII recombinants complets, ct pcut-être de douze à quinze ans pour les variants du facteur VIII. Ces variants bénéficient des connaissances Ainsi, ils ne contiennent pas de domaine B (Tableau II, [31, 32]), ce qui peut expliquer qu'ils sont plus facilement sécrétés par les cellules du fait d'un moindre degré de glycosylation de la protéine [4].

Certains de ces variants sont bicaténaires et d'autres monocaténaires (Tableau II), et les modes de purification varient en fonction des contaminants apportés par le milieu de culture cellulaire.

Cette multiplicité de nouveaux facteurs antihémophiliques $\mathrm{A}$ potentiels reflète la demande d'un produit thérapeutique efficace et sûr.

L'efficacité du facteur VIII recombinant complet a été montrée chez de nombreux patients, notamment chez des hémophiles sévères. Les essais cliniques multicentriques ont montré que ce produit recombinant était bien toléré ct aussi cfficace que le facteur VIII plasmatique. Par ailleurs, des études réalisées in vitro et in vivo chez des chiens hémophiles ont prouvé également l'efficacité des facteurs VIII délétés de la région B. Cependant, les risques potentiels d'immunisation contre ces variants, liés notamment à la présence de néoantigènes, sont actuellement évalués dans différents modèles animaux et lors des premiers essais cliniques. A terme, les outils de la biologie moléculaire et les biotechnologics auront façonné un ou plusieurs facteurs antihémophiliques A plus efficaces et plus sûrs sur la relation structure-fonction.

\section{Summary}

Recombinant factor VIII (antihemophilic A) : structurefunction relationship

Factor VIII is a glycoprotcin which acts as a cofactor in the blood coagulation pathway. Sequence modifications or a deficiency in factor VIII cause a serious bleeding disorder called haemophilia A. Gene cloning has greatly contributed to the understanding of the factor VIII structure and function. The primary sequence, deduced from the human cDNA, predicts a mature polypeptide of 2332 aminoacids which exhibits three domains arranged as follows : A1-A2-B-A3-C1-C2. Factor VIII is present in plasma as a complex corresponding to the association, by a divalent ion, of a $210 \mathrm{kDa}$ heavy chain (A1-A2-B) to a $80 \mathrm{kDa}$ light chain (A3-C1-C2). Cleavages of the $\mathrm{B}$ domain lead to different heterodimers of Mr ranging from 210-80 to $90-80 \mathrm{kDa}$. Factor VIII acts in the blood coagulation pathway in its activated or inactivated forms after proteolysis by thrombin or activated protein C, respectively. Site-directed mutagenesis has allowed an analysis of the structural requirements for factor VIII function. Thrombin activation corresponds to proteolysis on the heavy and light chains after the Arg 372 and 1689, respectively, to generate the activated trimer A1/A2/A3-C1-C2. Dissociation of the A2 domain from this complex leads to factor VIII inactivation. By contrast, inactivation with the activated protein $\mathrm{C}$ corresponds to the proteolysis of the heavy chain after Arg 336 to generate the A1/A3-C1-C2 dimer. Considering these structural requirements and that the $\mathrm{B}$ region is dispensable for procoagulant activity, different variants of factor VIII comprising partial or complete delctions of the $\mathrm{B}$ domain have been constructed. These variants, which are better secreted than the complete protein, are active to restore coagulation in vitro and in vivo in hemophilic dogs. The clinical investigations have shown that the completc recombinant factor VIII is efficacious for treatment of haemophilia A. 